\title{
Publication of maps and plans of Jewish settlement from the first half of the 18th century
}

\author{
Šárka STEINOVÁ $^{\mathrm{a}}$, Jiř́i DROZDA ${ }^{\mathrm{b}, *}$, Filip PAULUS ${ }^{\mathrm{a}}$, Ondřej BÖHM $^{\mathrm{b}}$ \\ ${ }^{a}$ National Archives Czech Republic, sarka.steinova@nacr.cz,filip.paulus@nacr.cz \\ ${ }^{b}$ Research Institute of Geodesy, Topography and Cartography,v.v.i., jiri.drozda@vugtk.cz, ondrej.bohm@vugtk.cz \\ * Corresponding author
}

\begin{abstract}
:
A unique set of maps and plans, showing in detail the Jewish settlement in the Czech Republic originating from the first half of the 18. century, was compiled and expertly handled by researchers of the Czech National Archives. The set consist of 98 plans and sketches produced on the basis of the Imperial Translocation Rescripts (1726). All maps and sketches have been produced within one year 1727 and depicted small towns and villages in some cases already extinct. During research large data base file was created with the aim of spatial identification of individual maps and plans, and objects that appear on them. In cooperation with the VÚGTK application has been developed to publish this unique map set through the Web application. The application allows viewing individual maps including the preserved archive materials, their overlap with other major cartographic sources, mainly with the Index Sketches of the Stable Cadastre dating from the first half of the 19th century. (The Index Sketches of the Stable Cadastre was one of the main sources used in the professional processing and spatial identification of individual maps.) The application also allows comparing with the current cartographic materials (aerial imagery, nowadays maps, GIS data etc.) The benefit of application is not only for research of Jewish settlement development in the Czech Republic but also for other research like urban planning and development of the countryside and its settlements.
\end{abstract}

Keywords: Jews, settlements plans, maps publication

\section{Introduction}

The plans and sketches of Jewish settlements in Bohemia in first half of $18^{\text {th }}$ century represent a unique testimony as to the shape of towns and villages at that time. In keeping with our long time efforts at VÚGTK to make available online old maps and related documents we created a web application showing the plans of Jewish settlements in the context of old and current map data.

\section{Source data}

These plans of Jewish settlements were created in the first half of $18^{\text {th }}$ century during a mapping of Jewish settlements in Czech Crown Lands initiated by the socalled Familiar Law of 1727 and subsequent imperial translocation rescripts. The result was a set of 98 plans and sketches capturing with varying degree of precision the state of the then settlements. In many cases these maps are the only such information preserved to present. (Roubík, F.,1931)

The plans show general picture of a given area with indicated positions of churches and Jewish dwellings, synagogues and cemeteries. Some of the plans include perspective views providing insight into original appearances of individual buildings. Other maps show ground plan and about $20 \%$ of the maps contain both perspective views and ground plans.

The drawings and ground plans are complemented by captions and descriptions placed either at the edges or inside the image area as space on the map sheet permitted. The textual part of most maps is in German with some in Latin or Czech. Some texts are written in a combination of Latin and Czech or Czech and German.

Dimensions of the maps vary from $25 \times 30 \mathrm{~cm}$ to $65 \times 45$ $\mathrm{cm}$ with some exceptions as small as $18 \times 12 \mathrm{~cm}$ or as large as $100 \times 37 \mathrm{~cm}$. Their scale where applicable is mostly in "Prague Loket" (ell) or "sáh" (provincial fathom) (Steinova et al. 2019).

The amount of information, workmanship and fidelity of the maps varies greatly. Some of them are simple sketches providing only basic overview of the situation while others are detailed works exhibiting great technical and artistic skill. The cause of these differences is diversity of authors. While some of them were trained professionals like surveyors, military engineers or urban builders other authors were town scribe or complete laymen without any cartographic expertise. 


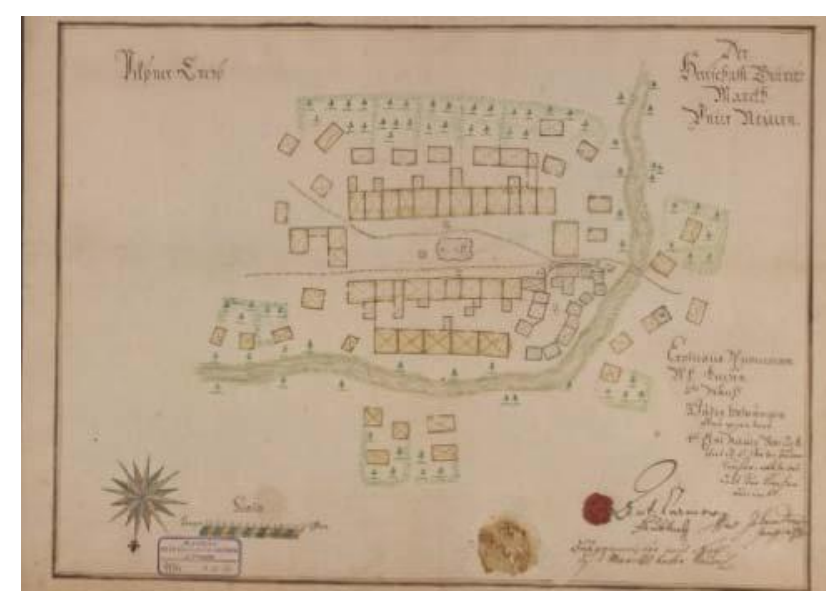

Figure 1. Example map from the set - hand-colored map of the town of Dolní Nýrsko from 1727 with a floor plan of the Church of the Fourteen St. Helpers. Scale 1:1294. National Archives of Prague, Collection of Maps and Charts, inv. no. 1174 , sign. A/XII/ $/ 16$

\subsection{Data Collection}

The maps had been stored at the registry of Old Czech Administration at first but later were moved to the Collection of the Old Administration thanks to the efforts of archivists Jan Josef Klauser and Kajetan Nadherny. Later still they were transferred to the Czech Archives, the Archives of the Ministry of the Interior, and later to the State's Central Archives and finally since 2002 they have been stored at the National Archives (the successor organization of the Archives of the Ministry of the Interior) (Steinova et al. 2019).

Data from the catalogs of the National Archives formed the basis of a database of information about the maps. These data were further expanded by additional information by authors of (Steinova et al. 2019) settlements typology, processing techniques, colouring, witness' names, seals and, most notably with relation with application discussed in this paper, coordinates of selected buildings and map scales.

Identification of buildings and features was done with the help of the Index Sketches of the Stable Cadastre and current aerial photography. Using these resources many current locations of buildings depicted on the maps can be identified. Still, some sites have changed significantly or completely disappeared. In these cases the maps are the only evidence of settlements in $18^{\text {th }}$ century in given areas.

\section{Publishing the maps online}

Publishing maps online is in general a multistep process. The steps are:

\section{- scanning}

- preparation for publication

$$
\begin{array}{ll}
\circ & \text { crop scans } \\
\circ & \text { georeference } \\
\circ & \text { transformation into appropriate format } \\
& \text { (tiles, zoomify, etc.) }
\end{array}
$$

- the actual publication - creation of web presentation or application

\subsection{Scanning}

First step to publishing maps online is to scan them. During this process the specifics of the work should be observed - the maps should be scanned with sufficient resolution and ideally on a precise scanner (Antoš et al., 2012) to preserve cartographic qualities of the original maps.

\subsection{Preparation}

The raw scans have to be prepared for publication with the exact steps varying based on method of publication. In general the scans have to be at least cropped to just the map sheet and transformed into a format suitable for publication on the internet i.e. a map layer served by an image server of choice.

Ideally this step would include georeferencing (i.e. associating maps with spatial location). However in our case not all of these maps can be georeferenced. Even ignoring the low cartographic quality of some of the maps in some cases the situation changed so much no suitable identical points can be found. Still all maps can be at least associated with specific town or village.

Based on the number of maps and their character the process of georeferencing can be very time consuming. It's necessary to collect a fair number of identical points and more importantly to have some cartographic and technical expertise to carry out the georeference. While there are tools available to help with this task they generally cannot produce results tailored to exact needs of a custom map application or online presentation.

It should be noted that georeferencing is not strictly required to publish maps online and even without it the maps can be displayed. However georeferencing greatly increases the usefulness of digital maps by allowing combining them with other map sources available via various standard protocols (WMS - Web Map Service, TMS - Tile Map Service, etc.).

\subsection{Application}

The last step is developing an online presentation. That can vary a lot in terms of provided features from simple presentation to full featured GIS (Geographic Information System). The application described in this paper falls somewhere in the middle. The goal was to not only put the scans of maps on display but also:

1. Provide supplemental interesting data.

2. Show the maps in context of other maps both contemporary and current when possible.

The supplemental data and map metadata were collected by researchers from the National Archives by exploring the maps and referencing archival documents.

To fulfil the second objective the application uses map sources created under other VÚGTK's grants (especially NAKI - Cartographic Sources as Cultural Heritage) and sources available from other providers (like ČÚZK, Bing Maps from Microsoft and OpenStreetMap).

The application presents an overview map of the Czech Republic with locations of the Jewish plans marked by 
icons. It's possible to choose the background map layer from several maps sources:

- 3rd Military Survey

- Special Maps of 3rd Military Survey

- current aerial map of the Czech Republic (sourced from ČÚZK [3])

- $\quad$ current topographic map (either from Bing Maps or OpenStreetMap)

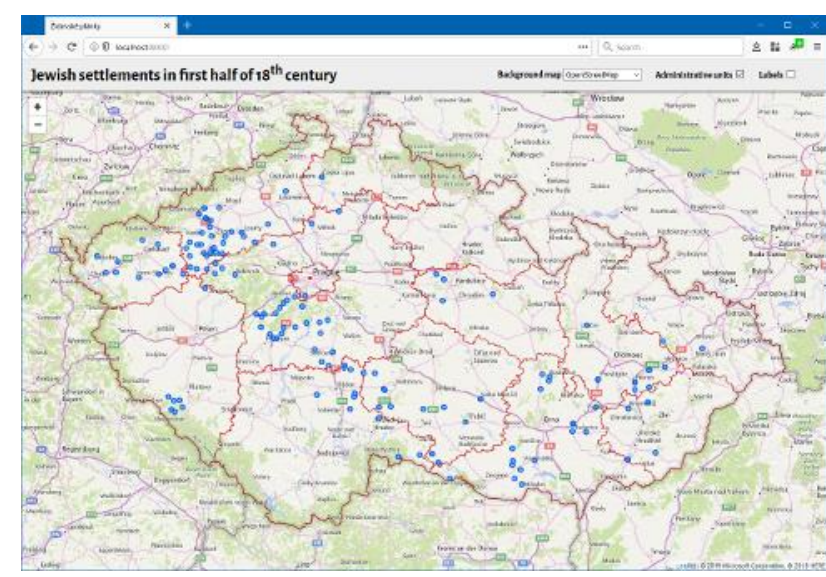

Figure 2. Initial view of the application. Blue circle markers show locations of Jewish settlements at the time of creation of the set of maps

Clicking on an icon representing one of the maps opens a detailed view of the map in question. This view displays the map in full resolution and shows relevant metadata. If the map is georeferenced users can again choose a background map (from the list above expanded by Index Sketches of Stable Cadastre), or opt to not use any background. Another option is to open one of the supplementary map layers in a side-by-side view to the old map. This works for both georeferenced and nongeoreferenced maps. Either of these detailed views allows comparing the Jewish settlement map with other map sources and exploring differences and changes over time in given settlements.

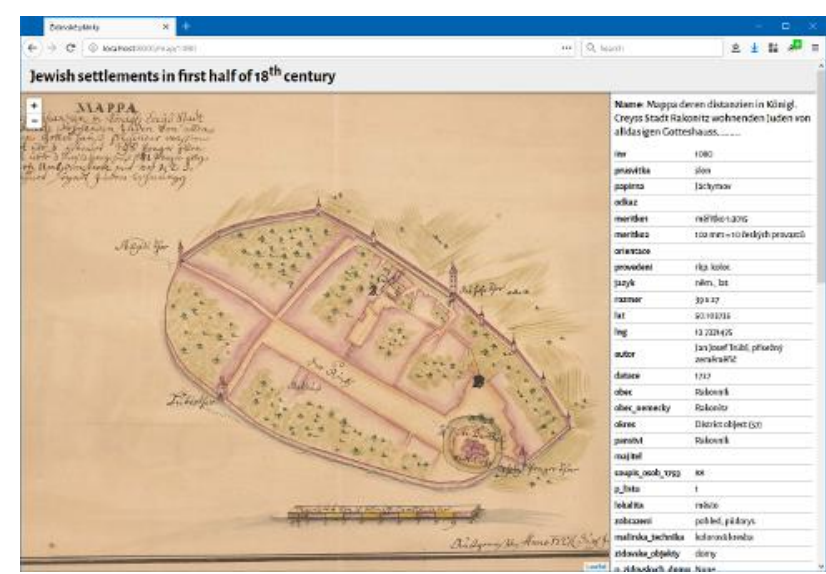

Figure 3. Detail view of map of town Rakovnik in 1727. National Archives of Prague, Collection of Maps and Charts, inv. no. 1080, sign. F/X/3. Main area shows the map scan in full resolution (zoomable) and right sidebar display metadata.

\subsection{Technologies}

The application's server side is written in Python using Django web framework and runs on an Apache web server. The client side uses the excellent Leaflet JavaScript library for displaying map data provided via Tile Map Service (TMS) (for georeferenced maps) or Zoomify protocol (for non-georeferenced maps).

Map metadata are stored in SQLite3 database. Given the dataset is comparatively small the database structure is extremely simple - a single table where each record ("row") holds information about one map.

\section{Conclusion}

The application described in this paper displays a unique source of details on the shape of towns and villages in early $18^{\text {th }}$ century Bohemia. Furthermore it demonstrates the advantages of web as a platform for publication of old maps and use of open standards - easy accessibility to a wide audience, detailed high fidelity and interactive presentation of source documents, possibility of displaying documents in context of other data sources and ability to combine various data sources together.

\section{References}

Roubík, F. (1931). Maps of municipalities in the Czech Republic, with an indication of the Jewish homes dating from 1727. In: Časopis Společnosti přátel starožitností českých 39, 1931, s. 49-68. Prague, the Czechoslovak Republic.

Antoš, F., Böhm, O., Talich, M. and Ambrožová K.: Cartographic sources as a cultural heritage. In: 4th International Symposium of the ICA Commisssion on the History of Cartography; Eötvös Loránd University, Budapest, Hungary, 28-29 June, 2012.

Steinová, Š., Drozda, J. and Paulus, F.: Jewish settlement plans in Bohemia from the first half of the 18th century. In: ICA Advances, 29th Cartographic Conference, 2019, Tokyo (accepted). 